\title{
Sasa quelpaertensis leaf extract regulates microbial dysbiosis by modulating the composition and diversity of the microbiota in dextran sulfate sodium- induced colitis mice
}

\author{
Yiseul Yeom ${ }^{1}$, Bong-Soo Kim², Se-Jae Kim³ and Yuri Kim ${ }^{1 *}$
}

\begin{abstract}
Background: Inflammatory bowel diseases (IBD) are related to a dysfunction of the mucosal immune system and they result from complex interactions between genetics and environmental factors, including lifestyle, diet, and the gut microbiome. Therefore, the effect of Sasa quelpaertensis leaf extract (SQE) on gut microbiota in a dextran sulfate sodium (DSS)-induced colitis mouse model was investigated with pyrosequencing of fecal samples.

Methods: Three groups of animals were examined: i) a control group, ii) a group that was received 2.5\% DSS in their drinking water for 7 days, followed by 7 days of untreated water, and then another 7 days of 2.5\% DSS in their drinking water, and iii) a group that was presupplemented with SQE (300 mg/kg body weight) by gavage for two weeks prior to the same DSS treatment schedule described in ii.

Results: SQE supplementation alleviated disease activity scores and shortened colon length compared to the other two groups. In the DSS group, the proportion of Bacteroidetes increased, whereas that the proportion of Firmicutes was decreased compared to the control group. SQE supplementation recovered the proportions of Firmicutes and Bacteroidetes back to control levels. Moreover, the diversity of microbiota in the SQE supplementation group higher than that of the DSS group.

Conclusion: SQE was found to protect mice from microbial dysbiosis associated with colitis by modulating the microbial composition and diversity of the microbiota present. These results provide valuable insight into microbiota-food component interactions in IBD.
\end{abstract}

Keywords: Sasa quelpaertensis leaf extract, Inflammatory bowel disease, Dextran sulfate sodium, Gut microbiota

\section{Background}

Inflammatory bowel diseases (IBD), including Crohn's disease (CD) and ulcerative colitis (UC), are chronic and recurrent inflammatory disorders with uncertain etiology [1]. IBD are generally accompanied by abdominal pain, weight loss, and diarrhea, and can result in complete obstruction of the gastrointestinal (GI) tract [2]. Worldwide, the incidence and prevalence of IBD have increased over

\footnotetext{
* Correspondence: yuri.kim@ewha.ac.kr

${ }^{1}$ Department of Nutritional Science and Food Management, Ewha Womans University, Seoul 03760, Republic of Korea

Full list of author information is available at the end of the article
}

the past few decades. To date, the precise pathogenesis of IBD remains unclear, although dysfunction of the immune system due to interactions between a host's response to microbial flora in the gut may be one of the main factors that contribute to these diseases [3].

Hunan gut microbiota consists of more than 100 trillion microorganisms, which is ten times more than the total number of human cells in the body [4]. In general, a fetus grows in a sterile environment in the uterus. Then, after birth, gut colonization starts rapidly and it is influenced by a variety of factors, including diet, antibiotics, and stress [5]. Gut microbiota have diverse and 
useful functions in energy balance, glucose metabolism, drug metabolism, and inflammation in a host [6]. However, when an imbalance in normal gut microbiota occurs, this is known as dysbiosis. Dysbiosis underlies the pathogenesis of numerous diseases, including IBD, colorectal cancer, and metabolic syndrome in connection with host metabolism $[7,8]$. For obese individuals, their intestinal microbiota contains a higher proportion of Firmicutes, and a lower proportion of Bacteriodetes compared to lean individuals $[9,10]$. Insulin sensitivity and plaque synthesis in blood vessels can also be altered by gut microbiota $[9,11]$. Furthermore, changes in the population and metabolism of the diverse bacteria population in a GI tract can affect systemic inflammation and the function of neurotransmitters in the brain $[12,13]$.

Gut microbiota plays a critical role in anti-inflammatory and immune-regulatory function, and thus, potentially represent an attractive IBD therapy. Various therapies that target restoration of the gut microbiota by altering their composition have been suggested, including fecal microbiota transplantation, probiotics, prebiotics, antibiotics, and dietary intervention. Recent interest in the dietary phytonutrients that are present in natural herbs has led to investigations of their potential impact on human health. For example, the polyphenols present in various natural herbs have been reported to modulate the composition and numbers of gut microbiota and to indirectly influence metabolism and the bioavailability of gut microbiota [14]. Another key benefit that has been found is an absence of undesirable side effects. Thus, gut microbiota may represent a potential therapeutic strategy for IBD and may help maintain intestinal function [15].

Sasa quelpaertensis Nakai is an edible dwarf bamboo grass that inhabits the area surrounding Mt Halla on Jeju Island in Korea. Its leaf extract has been reported to mediate various health promoting properties, including anti-inflammation, anti-cancer effects, and anti-obesity effect [16-18]. Sasa quelpaertensis leaves extract (SQE) is a mixture of polysaccharides, amino acids, and polyphenols, including $p$-coumaric acid and tricin, and has exhibited anti-inflammatory and anti-obesity effects [19, 20]. In particular, SQE has been found to mediate antiinflammatory effects by regulating inflammatory mediators such as nitric oxide, tumor necrosis factor $\alpha$, and COX-2 both in vivo and in vitro [17]. However, there is limited evidence regarding the effect of SQE on gut microbiota during inflammation.

Therefore, in the present study, the ability of SQE to regulate inflammation by modulating microbial composition in a dextran sulfate sodium (DSS)-induced colitis animal model was evaluated using highthroughput sequencing of the $16 \mathrm{~S}$ ribosomal rRNA (rRNA) gene.

\section{Methods}

\section{Preparation of SQE}

SQE was prepared as previously described [17]. Sasa quelpaertensis Nakai voucher specimen has been deposited in a publicly available herbarium name as HALLA ARBORETUM HERBARIUM and deposit number is HA006630. Briefly, collected Sasa quelpaertensis Nakai leaves $(1 \mathrm{~kg})$ were collected from Mt. Halla on Jeju Island, South Korea and were washed twice with deionized water. The leaves were then dried and extracted with $70 \%$ ethanol for $48 \mathrm{~h}$ at room temperature. After the SQE was filtered, it was concentrated with a rotary evaporator under reduced pressure and freeze-dried. The resulting SQE extract was crushed into a powder and stored at $-20{ }^{\circ} \mathrm{C}$ until needed. Previously, we have reported that $p$-coumaric acid and tricin were two major bioactive compounds in SQE and determined the concentrations of these compounds using high performance liquid chromatography (HPLC) 2695 Alliance System (Waters Corp., Mildford, MA, USA). The concentrations of each $p$-coumaric acid and tricin were 1.13 and $0.82 \mathrm{mg} / \mathrm{g}$ [17].

\section{Induction of DSS-induced colitis in mice}

Five-week-old male C57BL/6 mice were purchased (Central Lab, Animal Inc., Seoul, Korea) and maintained under standard laboratory conditions: $22 \pm 2{ }^{\circ} \mathrm{C}, 50 \pm$ $5 \%$ humidity, and a $12 \mathrm{~h} / 12 \mathrm{~h}$ light/dark cycles. Animals received a modified American Institute of Nutrition (AIN)-93G pellet diet (Unifaith, Inc., Seoul, Korea). Diet composition was provided in Table 1. To confirm their health status, all mice were housed for 1 week before being randomized into three groups ( $n=6 /$ group).

The three experimental groups included: i) mice receiving a standard diet and normal drinking water (control), ii) mice receiving 2.5\% DSS (DSS), and iii) mice receiving DSS + SQE [300 mg/kg body weight (b.w.)] (SQE). The DSS and

Table 1 Dietary composition for the experiment

\begin{tabular}{ll}
\hline Ingredients (g) & $\mathrm{g} / \mathrm{kg} \mathrm{diet}$ \\
\hline Casein, lactic & 200 \\
L-cystein & 3 \\
Corn starch & 397.5 \\
Maltodextrin & 132 \\
Sucrose & 100 \\
Cellulose & 50 \\
Soybean Oil & 70 \\
Mineral mix, AlN-93G ${ }^{\text {a) }}$ & 35 \\
Vitamin mix, AIN-93G & 10 \\
Cholin Bitartrate & 2.5 \\
t-butylhydroquinone & 0.014 \\
\hline
\end{tabular}

a) Mineral mixture and vitamin mixture were prepared according to AIN-93G diet 
SQE groups received 2.5\% DSS (molecular weight: 36 - 50 $\mathrm{kDa}$; MP Biomedicals, Costa Mesa, CA, USA) in their drinking water for $7 \mathrm{~d}$, followed by $7 \mathrm{~d}$ of untreated drinking water, and then another $7 \mathrm{~d}$ of $2.5 \%$ DSS in their drinking water. The SQE group mice received a daily oral dose of SQE for $14 \mathrm{~d}$ prior to DSS treatment. During the experimental period, body weight and diet intake were recorded twice a week. After five weeks, all of the mice were sacrificed. Animal care and experimental protocols for this study were approved by the Animal Care and Use Committee of Ewha Womans University (IACUC approval no: IACUC 14-070).

\section{Disease activity index (DAl)}

DAI scoring was measured from the start of DSS administration until the end of the experimental period as described previously [17]. DAI scores were determined based on weight loss, stool consistency, and fecal bleeding. Stool consistency was evaluated according to the presence of loose feces and watery diarrhea. Fecal bleeding was scored as normal, slightly bloody, and blood in whole colon compared to the control group.

\section{Genomic DNA extraction}

To analyze gut microbiota analysis, fecal samples were collected at $19 \mathrm{~d}$ after the start of the DSS treatment. Metagenomic DNA was extracted with Fast DNA SPIN Kits (MP BIO, Santa Ana, CA, USA), according to the manufacturer's instructions. The resulting metagenomic DNA samples were dissolved in $50 \mu \mathrm{l}$ of elution buffer and stored at $-20{ }^{\circ} \mathrm{C}$ until needed. DNA concentrations were determined based on optimal density value obtained at $260 \mathrm{~nm}$. Sample purity was determined based on the ratio of the absorbance values obtained at 260 $\mathrm{nm}$ and $280 \mathrm{~nm}$.

\section{Pyrosequencing analysis of gut microbiota based on the 16S rRNA gene}

The 16S rRNA gene (targeted V1-V3 regions) was amplified from the extracted DNA using barcoded primers (27 F and 518R). The resulting PCR products were confirmed by gel electrophoresis and purified. Sequencing of the amplicons was conducted using a Roche/454 GS Junior system (ChunLab, Inc., Seoul, Korea). Data analysis was performed according to previously described method [21]. Each sample was sorted according to a unique barcode. Low quality reads (average quality score $<25$ or read length $<300 \mathrm{bp}$ ) did not undergo further analysis. The primer sequences were trimmed and clustered for correcting sequencing errors. The taxonomic positions of the representative sequences for each cluster were identified using the EzTaxon-e database [22]. Chimeric sequences were removed using the UCHIME program [23] and the diversity indices were calculated with the Mothur program [24]. The pyrosequences presented in this study are available in the EMBL SRA database under the study PRJEB13815 (http://www.ebi.ac.uk/ ena/data/view/PRJEB13815). The operational taxonomic unit (OTUs) were mathematically defined as having a $3 \%$ sequence distance (e.g. 97\% similarity). Diversity and richness were calculated using the Cluster Database at High Identity with Tolerance (CD-HIT). Alpha diversity indices such as Chao1 and Shannon diversity were used to estimate species richness using the Mothur program and the matrix of Fast UniFrac. Principal coordinate analysis (PCoA) was used to represent the relationships between samples based on calculations of Jaccard abundance similarity and Bray-Curtis similarity $[24,25]$.

\section{Statistical analysis}

Statistical analyses were performed using GraphPad PRISM software (GraphPad Software, SanDiego, CA, USA). Data presented are the mean \pm standard error of the mean (SEM) for each group. For multiple comparisons, one-way analysis of variance (ANOVA) with Newman-Keuls's post-hoc test was used. A $P$-value less than 0.05 was considered statistically significant.

\section{Result}

\section{DAI and colon length}

DAI scores were significantly increased up to day 5 and peaked on day 19 in the DSS group compared to the control group (Fig. 1a). The increase in DAI values was based on the observed incidence of diarrhea, weight loss, and bloody stools. In contrast, the DAI scores of the SQE group were significantly attenuated by $61.9 \%$ at day 5 and by $77.4 \%$ at day 19 compared to the DSS group $(p<0.05$ in each case). Moreover, the DAI score for the SQE group was comparable to that of the control group.

Since severity of DSS-induced colitis was found to be associated with a shorter colon length [26], whole colon tissues were isolated from each group and their lengths were and compared. The mean colon length of the DSS group was $27 \%$ shorter than the mean colon length of the control group, and SQE supplementation significantly attenuated shortening of the colon compared with the DSS group (Fig. 1b).

\section{OTUs and diversity estimates for fecal microbiota}

The average numbers of analyzed sequence reads were $5310 \pm 1519$ for the control group and $4722 \pm 1092$ for the DSS group, and $4744 \pm 1092$ for the SQE group (Table 2). The Good's coverages of all the samples were greater than 0.97 . The number of observed OTUs was $444.83 \pm 66.84$ for the control group, $256.33 \pm 65.64$ for the DSS group, and $404.17 \pm 178.21$ for the SQE group. The number of observed OTUs was significantly lower for the DSS group compared with the control group by 

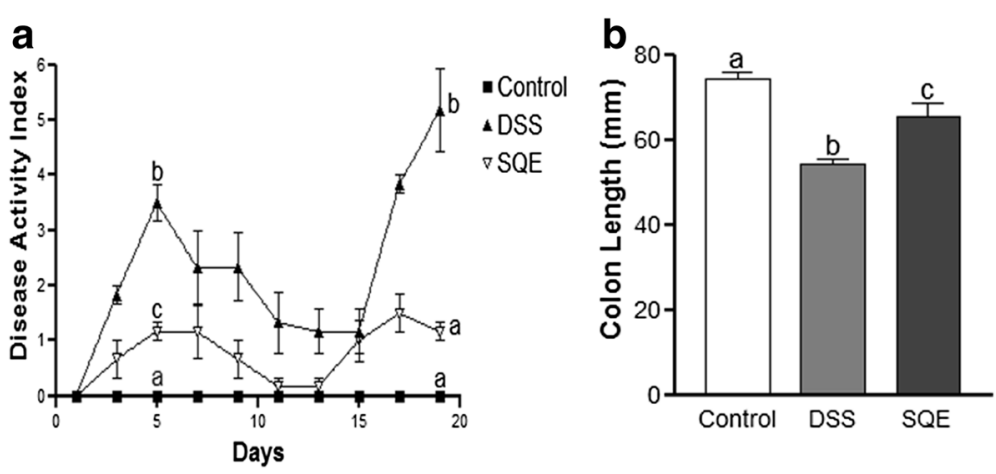

Fig. 1 DAI scores and colon length in the DSS-induced colitis. a DAI values were evaluated based on observed changes and scoring of body weight loss, stool consistency, and fecal bleeding. b, Colon length was measured and compared among the control, DSS, and SQE groups. Data shown are the means \pm SEM and were analyzed by one-way ANOVA and Newman-Keuls's post hoc test $(p<0.05)$; $n=6$ mice per group

42.4\%, while the number of OTUs in the SQE group tended to be greater than the number of OTUs for the DSS group (57.7\%). Similarly, the number of estimated OTUs (Chao1) in the DSS group was significantly lower than those for the control group $(p<0.05,44.3 \%)$, while those for the SQE group were higher compared to the DSS group $(p<0.05,62.2 \%)$. The Shannon diversity indices for the control group were also significantly higher than those for the DSS group, yet were similar to those of the SQE group. Taken together, these results indicated that the diversity of gut microbiota in the DSS group were more diverse than the gut microbiota of the control and SQE groups.

\section{Comparison of gut microbiota}

To compare microbial community members among the three groups, clustering patterns based on a weighed pairwise Fast UniFrac analysis was determined (Fig. 2). The gut microbiota obtained from the DSS group was distinct from those of the control and SQE group, and the gut microbiota of the SQE group were closer to the control group in PCoA plot.

\section{Comparison of gut microbiota composition with pyrosequencing}

To analyze gut microbiota, fecal samples were collected at day $19 \mathrm{~d}$ after the start of the DSS treatment and they were analyzed using pyrosequencing. Differences in the microbiota among the groups were compared at the phylum level (Fig. 3). DSS treatment greatly increased the levels of Bacteroidetes by $44.9 \%$, and decreased the levels of Firmicutes by $34.4 \%$ compared to the control group (Fig. 3a and b). Correspondingly, the ratio of Bacteroidetes to Firmicutes in the gut microbiota was higher for the DSS group compared to the control group (Fig. 3c). However, following SQE supplementation, the proportions of Bacteroidetes and Firmicutes returned to control levels. Moreover, the ratio of Bacteroidetes to Firmicutes decreased following SQE supplementation. In contrast, the levels of Proteobacteria and Deferribacteres did not significantly different among the three groups.

Gut microbiota were also compared at the class by heatmap analysis (Fig. 4a). The bacteria were divided into a major class and a minor class (representing $<10 \%$ of the total proportion). Clostridia, Bacteroidia, and Erysipelotrichi constituted the major class of bacteria detected, whereas Deltaproteobacteria, Deferribacteres_c, Gammaproteobacteria, Verrucomicrobiae, and Betaproteobacteria constituted the minor class of bacteria. The proportion of Bacteroidia and Gammaproteobacteria were $50.3 \%$ and $4.1 \%$ in the DSS group, while Clostridia was decreased by $62.2 \%$ in the DSS group compared with the control group (Fig. $4 \mathrm{~b}$ and c). In the SQE group, the proportion of Clostridia was more than two

Table 2 Summary of diversity indices obtained from pyrosequencing results

\begin{tabular}{lccc}
\hline & Control & DSS & SQE \\
\hline Analyzed sequence reads (avg.) & $5310 \pm 1519$ & $4722 \pm 1092$ & $4744 \pm 1096$ \\
Goods Coverage & $0.97 \pm 0.01$ & $0.98 \pm 0.01$ & $0.97 \pm 0.01$ \\
Observed OTUs & $444.83 \pm 66.84^{\mathrm{a}}$ & $256.33 \pm 65.64^{\mathrm{b}}$ & $404.17 \pm 178.21^{\mathrm{ab}}$ \\
Chaol estimators & $657.94 \pm 91.98^{\mathrm{a}}$ & $366.26 \pm 109.76^{\mathrm{b}}$ & $594.05 \pm 259.98^{\mathrm{a}}$ \\
Shannon diversity index & $4.75 \pm 0.19^{\mathrm{a}}$ & $3.96 \pm 0.31^{\mathrm{b}}$ & $4.52 \pm 0.69^{\mathrm{a}}$ \\
\hline
\end{tabular}

Values are mean \pm SD

Significantly different by one - way ANOVA and Newman-Keuls's post hoc test among the three groups $(p<0.05) ; n=6$ mice per group

${ }^{a b c}$ For a given column, data not sharing a common superscript letter significantly differ 


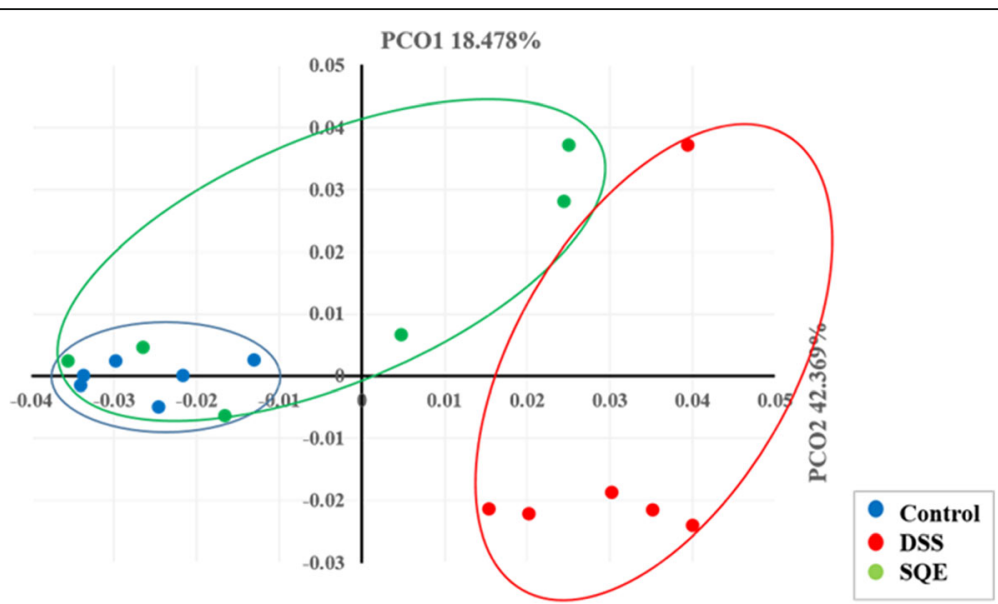

Fig. 2 Principal coordinate analysis (PCOA) plot. PCoA was used to determine clustering patterns among the control, DSS, and SQE groups $(n=6$ mice/group). Similarities between the communities were calculated by employing Fast UniFrac analysis

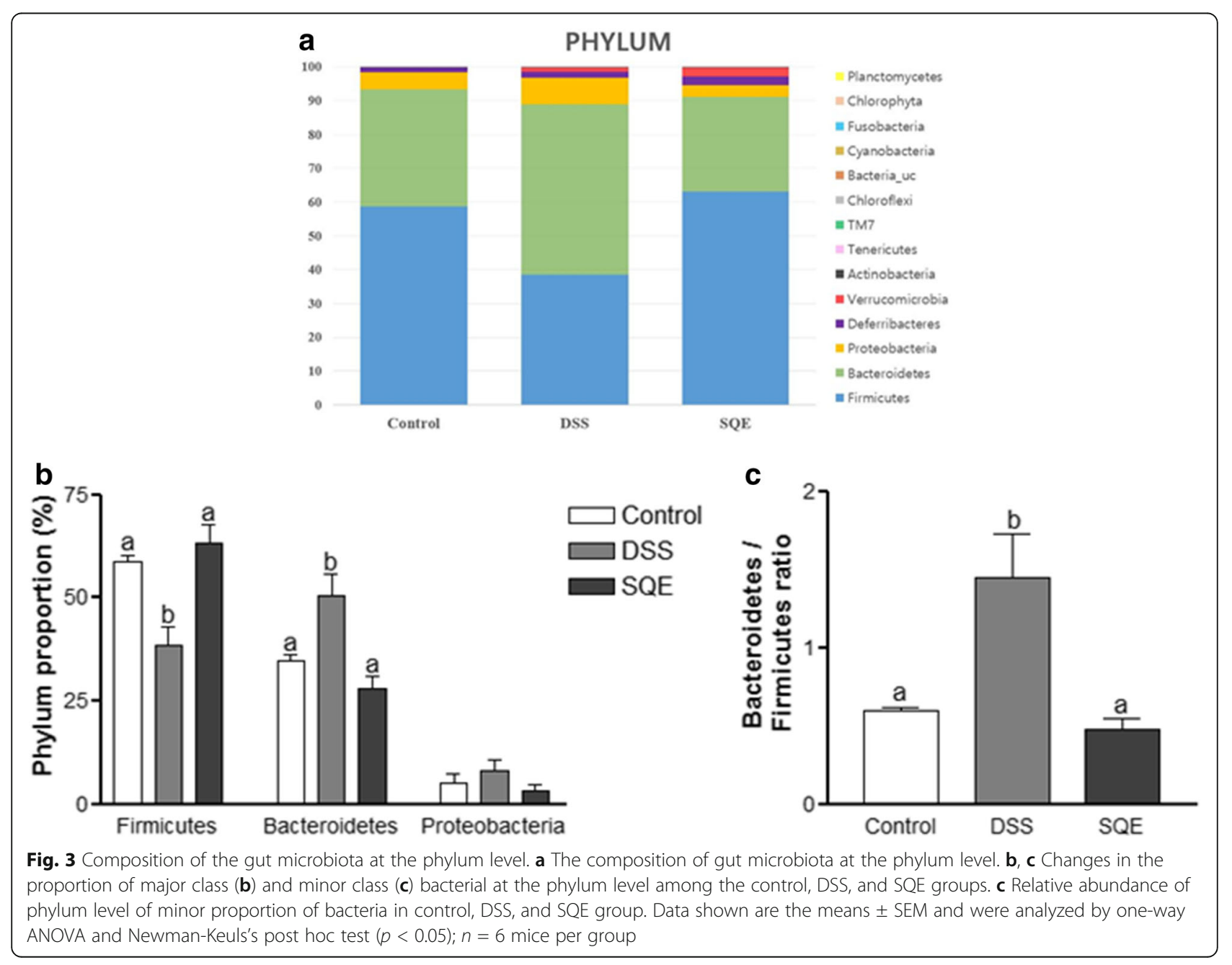




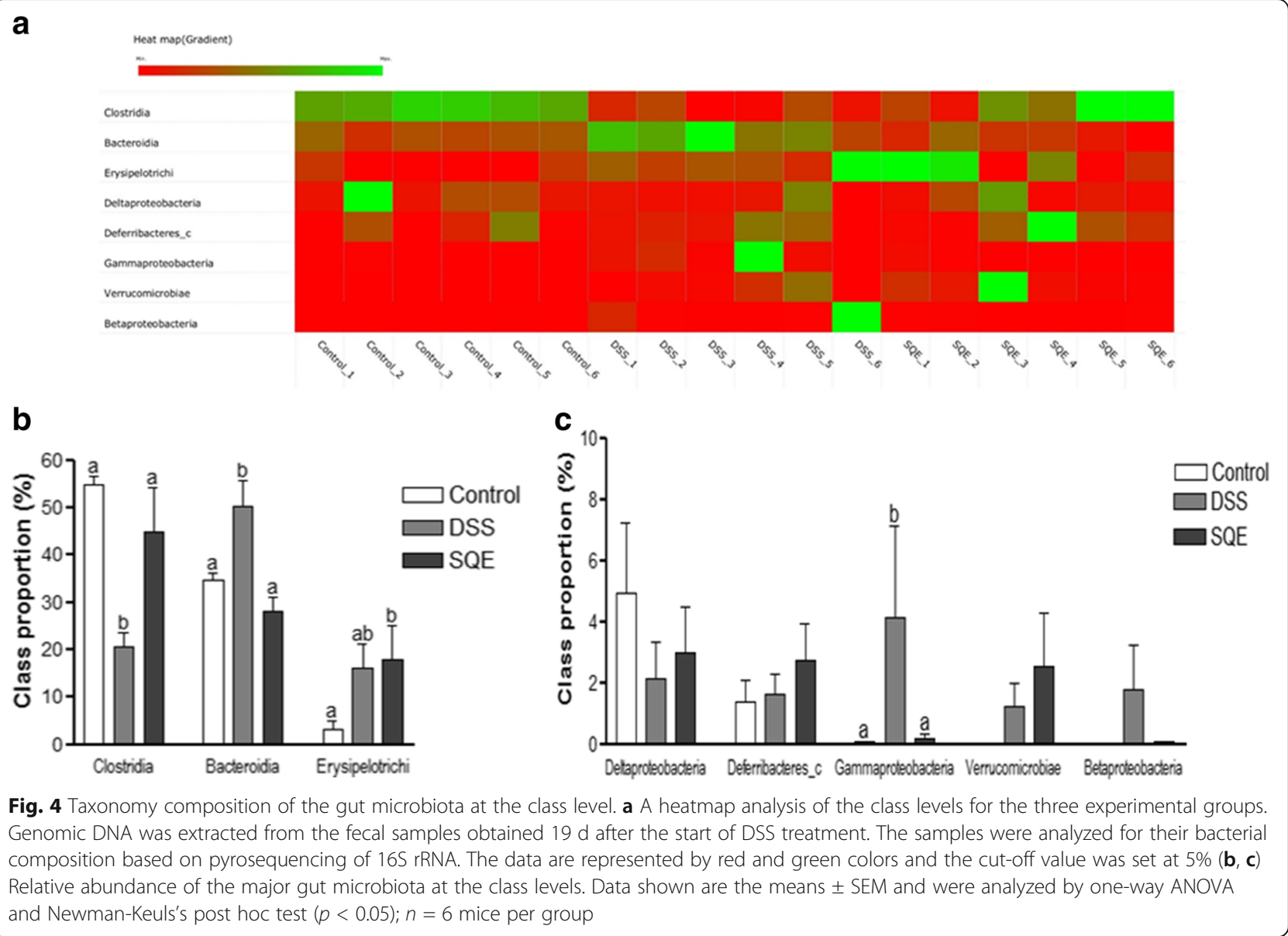

times higher, and the proportion of Bacteroidia and Gammaproteobacteria were significantly lower (83.6\%), compared to the DSS group. Moreover, both Bacteroidia and Gammaproteobacteria almost recovered to control levels.

Colitis led to the dysbiosis of the intestinal microbiota in the DSS treated mice at the family level, similar to the observations made at the phylum and class levels. When differences in the microbiota at the family level were compared (Fig. 5a). Lachnospiraceae, Bacteroidaceae, and Ruminococcaceae were found to be the dominant bacteria in all three group. DSS treatment decreased the proportions of Lachnospiraceae (68.4\%) and Ruminococcaceae (57.8\%), and increased the proportion of Bacteroidaceae two-fold compared to the control group (Fig. 5b). With SQE supplementation, the proportion of all three bacteria returned to the levels of control group. When the bacteria were divided into a major family and a minor family of bacteria, Lachnospiraceae, Bacteroidaceae, and Ruminococcaceae constituted the are major family of bacteria, while Coprobacillus, Prevotellaceae, and Enterobacteriaceae constituted the minor family of bacteria (representing less than 10\% of the total proporation). Among the minor bacteria, the proportion of Coprobacillus, and Enterobacteriaceae greatly increased following DSS treatment compared with the control group, and these increases were suppressed following SQE supplementation (Fig. 5c). In contrast, the abundance of Prevotellaceae and Streptococcaceae did not significantly differ among the three groups.

At the genus level, the proportion of Clostridium, Bacteroides, and Enterobacter significantly increased following DSS treatment compared to the control group, yet they decreased to control levels following SQE supplementation (Table 3). In contrast, the proportion of Hungarella and Alistipes significantly decreased following DSS treatment, while SQE supplementation tended to increase the proportion of these bacteria. At the species level, the proportion of Bacteroides acidifaciens $(p<0.001)$, Clostridium cocleatum $(p<0.001)$, and unclassified Bacteroides $(p<0.01)$ were significantly higher in the DSS group compared to the control group. However, following SQE supplementation, the proportion of these bacteria decreased back to the proportions observed in the control group (Table 4). These results suggest that SQE supplementation attenuates intestinal 


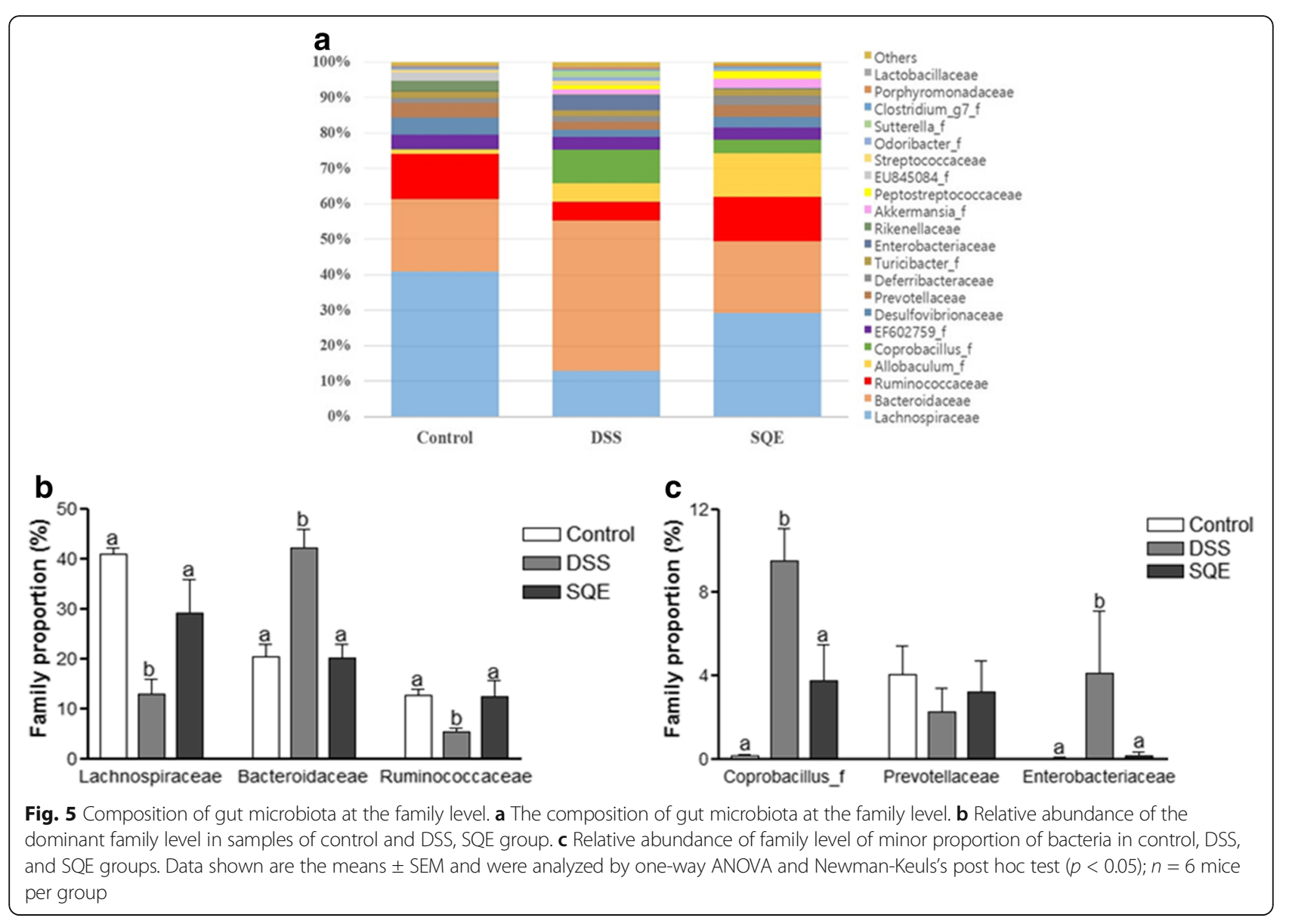

bacteria dysbiosis by regulating the bacteria compositional changes in bacteria that are associated with DSSinduced colitis in mice.

\section{Discussion}

The human gut contains a large population of diverse and complex enteric microbiota. Tremendous changes in the diversity and composition of this community, as well as the metabolic function of the gut microbiota, have been related to $\operatorname{IBD}[27,28]$. In particular, gut microbiota have been identified as a critical factor in IBD. Correspondingly, short-term antibiotic treatment for IBD patients have been used to suppress intestinal inflammation [29, 30]. Using murine model in gut microbiota study has been allowed functional and metabolic research on host-microbe interactions, and has brought more insights into the pathological mechanisms of IBD [31]. In colitis mouse model, the major gut microbiota shifted and gut bacterial diversity was reduced similar to those found in human IBD [32, 33].

Previously, it was reported that SQE treatment modulated the levels of proinflammatory markers, while also regulated the activation of nuclear factor $\mathrm{kB}$ and oxidative stress, in animal models of DSS-induced colitis [17, 34]. In the present study, the goal was to understand the effect of SQE on dysbiosis of microbiota in DSS-induced colitis. Therefore, overall differences in the microbial community, as well as modifications of microbiota composition after SQE treatment were investigated by using barcoded pyrosequencing of the $16 \mathrm{~S}$ rRNA gene. The results obtained demonstrate that the microbial community profiles of the experimental groups examined were altered by DSS treatment, and dysbiosis of gut microbiota was improved with SQE supplementation.

In animal models of IBD, DAI value and colonic length are key indicators for evaluating the severity of colitis $[35,36]$. Consistent with the results of a previous study [17], SQE supplementation attenuated the severity of colitis by lowering the DAI value and extending the length of the colon. In contrast, changes in the colon epithelium and higher DAI values characterized in the DSS group compared with the control group,

Modification to the composition of a microbial community may involve changes in diversity and in bacterial metabolism. Furthermore, an imbalance between obligate anaerobic bacteria and facultative anaerobic bacteria can occur, and this is related to the inflammation process $[37,38]$. For example, Ott et al. reported that a 
Table 3 Composition of fecal microbiota in DSS - induced colitis mouse model ${ }^{c}$

\begin{tabular}{|c|c|c|c|c|}
\hline Phylum & Genus & Control & DSS & SQE \\
\hline \multirow[t]{10}{*}{ Firmicutes } & Pseudoflavonifractor & $4.83 \pm 0.82$ & $2.71 \pm 1.32$ & $6.19 \pm 3.93$ \\
\hline & Clostridium_g6 & $0.17 \pm 0.13^{a}$ & $9.49 \pm 3.85^{b}$ & $3.75 \pm 4.23^{a}$ \\
\hline & Acetatifactor & $1.48 \pm 1.42$ & $1.31 \pm 1.30$ & $4.78 \pm 3.55$ \\
\hline & Oscillibacter & $1.68 \pm 0.75^{\mathrm{a}}$ & $1.12 \pm 0.76^{a}$ & $4.44 \pm 2.68^{b}$ \\
\hline & Hungatella & $3.56 \pm 1.07^{a}$ & $0.82 \pm 0.69^{b}$ & $1.50 \pm 1.28^{b}$ \\
\hline & Turicibacter & $1.72 \pm 3.57$ & $1.61 \pm 1.73$ & $1.73 \pm 2.48$ \\
\hline & Clostridium_g21 & $1.51 \pm 0.59$ & $0.75 \pm 0.44$ & $1.78 \pm 1.44$ \\
\hline & Romboutsia & $0 \pm 0$ & $1.20 \pm 2.93$ & $2.09 \pm 4.35$ \\
\hline & Lachnospiraceae_uc & $1.41 \pm 0.47$ & $0.25 \pm 0.29$ & $1.47 \pm 1.75$ \\
\hline & Roseburia & $0.17 \pm 0.08$ & $0.11 \pm 0.07$ & $0.24 \pm 0.17$ \\
\hline \multirow[t]{3}{*}{ Bacteroidetes } & Bacteroides & $20.23 \pm 6.55^{a}$ & $41.64 \pm 8.25^{b}$ & $20.10 \pm 6.43^{a}$ \\
\hline & Alloprevotella & $4.07 \pm 3.37$ & $2.20 \pm 2.76$ & $3.19 \pm 3.61$ \\
\hline & Alistipes & $3.11 \pm 1.12^{\mathrm{a}}$ & $0.31 \pm 0.23^{b}$ & $0.30 \pm 0.16^{b}$ \\
\hline \multirow[t]{2}{*}{ Proteobacteria } & Enterobacter & $0.02 \pm 0.03^{a}$ & $3.94 \pm 7.40^{b}$ & $0.04 \pm 0.05^{\mathrm{a}}$ \\
\hline & Parasutterella & $0.01 \pm 0.01$ & $1.78 \pm 3.55$ & $0.04 \pm 0.06$ \\
\hline Deferribacteres & Mucispirillum & $1.38 \pm 1.66$ & $1.64 \pm 1.56$ & $2.72 \pm 3.00$ \\
\hline
\end{tabular}

${ }^{c}$ Cut-off: 1.0

Values are mean \pm SD

Significantly different by one - way ANOVA and Newman-Keuls's post hoc test among the three groups $(p<0.05) ; n=6$ mice per group

${ }^{\mathrm{ab}}$ For a given column, data not sharing a common superscript letter significantly differ

microbial shift due to an increased in gram-negative bacteria accompanied a reduction in bacterial diversity in IBD patients, and this led to abnormalities in the inflammatory process [39]. In the present study, the analysis of various alpha diversity indices indicated that a reduction in bacterial diversity occurred in the DSS group compared to the control and SQE groups. In addition, the gut microbial communities of the DSS group were characterized by a

Table 4 Species level bacteria proportion

\begin{tabular}{lcll}
\hline $\begin{array}{l}\text { Group } \\
\text { Species }\end{array}$ & Control & DSS & SQE \\
\hline Bacteroides acidifaciens & $0.48 \pm 0.41^{\mathrm{a}}$ & $18.54 \pm 9.75^{\mathrm{b}}$ & $9.53 \pm 6.55^{\mathrm{a}}$ \\
Bacteroides sartorii & $7.03 \pm 10.51^{\mathrm{a}}$ & $6.22 \pm 8.79$ & $0.19 \pm 0.19$ \\
Clostridium cocleatum & $0.17 \pm 0.13^{\mathrm{a}}$ & $9.43 \pm 3.84^{\mathrm{b}}$ & $3.73 \pm 4.21^{\mathrm{a}}$ \\
Mucispirillum schaedleri & $1.38 \pm 1.66$ & $1.64 \pm 1.56$ & $2.72 \pm 2.98$ \\
Enterobacter xiangfangensis & $0.01 \pm 0.02$ & $3.38 \pm 6.37$ & $0.04 \pm 0.05$ \\
Akkermansia muciniphila & $0 \pm 0$ & $1.24 \pm 1.84$ & $2.51 \pm 4.24$ \\
Romboutsia ilealis & $0 \pm 0$ & $1.17 \pm 2.87$ & $2.08 \pm 4.32$ \\
Lachnospiraceae_uc_s & $1.41 \pm 0.47$ & $0.25 \pm 0.29$ & $1.47 \pm 1.75$ \\
Bacteroides_uc & $0.30 \pm 0.09^{\mathrm{a}}$ & $1.85 \pm 1.18^{\mathrm{b}}$ & $0.73 \pm 0.38^{\mathrm{a}}$ \\
Butyricimonas virosa & $0.45 \pm 0.17$ & $0.47 \pm 0.30$ & $0.38 \pm 0.19$ \\
Ruminococcaceae_uc_s & $0.54 \pm 0.49$ & $0.07 \pm 0.06$ & $0.27 \pm 0.23$ \\
Lactococcus lactis subsp & $0.41 \pm 0.32$ & $0.23 \pm 0.19$ & $0.15 \pm 0.11$ \\
\hline Values aremean \pm SD & & &
\end{tabular}

Values are mean \pm SD

Significantly different by one - way ANOVA and Newman-Keuls's post hoc test among the three groups $(p<0.05) ; n=6$ mice per group

${ }^{\mathrm{ab}}$ For a given column, data not sharing a common superscript letter significantly differ clustered distance to the control group. The latter result is consistent with the results of previous studies where microbial divergence manifested as relative abundance shift in cases of IBD [39, 40]. However, in the present study, SQE supplementation recovered the bacterial diversity of the gut and greater clustering of the gut microbial communities close to the control group was observed compared to the DSS group. Taken together, these results suggest that SQE may help the gut microbiota to maintain their composition, community, microbial evenness, and richness.

Interactions between gut microbiota and the host immune system play an important role in the development of a host's immune system [41]. Generally, the composition of gut microbiota remains stable during adulthood, and it can undergo dynamic changes in response to environmental stresses or diet. Such alterations in composition may influence health or disease risk [42]. The taxonomic compositions of the gut microbiota in humans is similar to that observed in mice at the phylum level [43]. Dysbiosis in patients with IBD has been characterized as an increase in the ratio of Bacteroidetes/Firmicutes $[28,44]$. In the present study, the ratio of Bacteroidetes/Firmicutes was significantly higher in the DSS group than in the control group, whereas this ratio in the SQE group was similar to that of the control group. It was also observed that the proportion of Firmicutes was significantly decreased following DSS treatment, yet the proportion recovered to control levels following SQE supplementation. The Firmicutes phylum 
modulates the $\mathrm{pH}$ of the colonic and inhibits the growth of pathogens by metabolizing short-chain fatty acids (SCFAs) and producing butyrate in the intestinal mucosa. Butyrate is a key energy source for epithelial cells of the colon and it suppresses pro-inflammatory cytokines in the gut [45]. At the class level, an increase in Bacteroidia (phylum Bacteroidetes) and Gammaproteobacteria, as well as a reduction in Clostridia (phylum Firmicutes) was observed in the DSS group compared to the control group. Gammaproteobacteria, a bacteria that can induce acute intestinal inflammation, was also significantly increased in the DSS group, thereby indicating that changes in intestinal permeability and induction of chronic systemic inflammation had occurred [46]. However, these changes in the composition of the microbial community were reduced in the SQE group compared to the DSS group, which suggested that SQE was able to regulate a gut microbial community by modulating gut inflammation.

In patients and experimental animal models with IBD, the relative abundance of Lachnospiraceae was reduced and the proportion of the Bacteroidaceae is relatively increased [47]. Lachnospiraceae plays an important role in fermenting SCFAs that derived from carbohydrates [48]. Another bacteria, Ruminococcaceae performs the first step in carbohydrate metabolism where hydrogen is consumed to butyrate. Microbial metabolisms of SCFAs is associated with gut motility and intestinal transit time, as well as with the function of histone deacetylases and the nervous system [49]. In the present study, the compositional abundances of Lachnospiraceae, Bacteroidaceae, and Ruminococcaceae, which mediate SCFA metabolism, were altered in mice of the DSS group. In contrast, microbial dysbiosis was improved in the SQE group compared with the DSS group. Previously, it was reported that SQE facilitated gut motility in the DSS-induced colitis mouse model [34], and this explains the role of SQE in the metabolisms of SCFAs and microbial composition related to intestinal function. Enterobacteriaceae (genus Enterobacter), obligate anaerobic bacteria for the metabolism of high energy nutrients, is present in greater number during inflammation [50]. In the present study, an increase in the proportion of Enterobacteriaceae was consistently detected in the DSS group compared with the control group, and this increase was blocked with administration of SQE.

As presented above, a strong connection between gut microbiota and the intestinal immune system has been observed. Among the various microbacteria, Clostridium (species Clostridium cocleatum) and Bacteroides (species Bacteroides acidifaciens, Bacteroides_uc) have been reported to induce the emission of regulatory $\mathrm{T}$ cells and to reduce intestinal inflammation [51]. In the present study, higher proportion of the Clostridium and Bacteroides were detected in the DSS group compared with to the control group, and these increase suggest that prevention of intestinal inflammation by specific groups of commensal obligate anaerobic bacteria may mediate direct protective effects for pathogens. Furthermore, the balance of microbial composition of species affects the bile acid metabolism in the colon. In particular, Enterobacter, Bacteroides, and Clostridium absorb dietary fats, facilitate lipid absorption, and maintain intestinal barrier function [52]. Consequently, dysbiosis resulting from intestinal inflammation can affect the function of bacteria and the other metabolic processes.

Many polyphenols contribute to important biological activities, including antioxidant, anticarcinogenic, and antimicrobial activities that are associated with pathological disease processes [53, 54]. In addition, most polyphenols are consumed and ingested, after being metabolized by gut microbiota, which leads to greater biological activity and increased bioavailability compared with their predecessors [55]. Furthermore, polyphenol intake may have a direct impact on the composition of gut microbiota and the functionality and the growth of certain bacterial species. For example, in the presence of phenolic compounds, the Firmicutes/Bacteroidetes ratio in the microbiota of obese individuals was found to be altered, and polyphenol-rich grape seed extract has been found to contain a higher proportion of Lactobacillus/Enterococcus bacteria [56, 57]. Several studies have also shown that natural herbs and polyphenols help to improve intestinal inflammation in colitis model [58]. SQE has shown beneficial effects on colitis in previous studies. Moreover, the bioactive component of SQE, tricin and $p$-coumaric acid, have exhibited antioxidant, anti-inflammatory, and anticancer effects which remain to be investigated in relation to gut microbiota $[17,18,20,34]$.

The identification of host and microbial interactions in IBD patients, as well as a greater understanding of the role of the microbiome and the changes in its composition that occur in the disease states of IBD, should lead to the development of highly effective and nontoxic targeted interventions to correct underlying abnormalities and induce sustained therapeutic responses. Currently, broad spectrum antibiotics, probiotics, and prebiotics are used to prevent and treat IBD [59]. The present results suggest a possible role for SQE and its various of polyphenols in the clinical treatments of IBD via regulation of gut microbiota dysbiosis and diversity. Moreover, the use of SQE would represent a natural therapeutic strategy for IBD patients. However, a clinical intervention trial is needed to confirm the present results in IBD patients, while additional research is needed to understand the relationship between dietary polyphenols and gut microbiota. 


\section{Conclusions}

The present study we demonstrated that DSS-induced colitis changed the diversity of the intestinal microbial composition and diversity led to an increase of inflammation in colon. However, when SQE was administered prior to the induction of colitis by DSS, microbial dysbiosis was reduced. These results increase our understanding of the important role that gut microbacteria have in maintaining intestinal homeostasis, and they also support the natural therapeutic potential of SQE for modulating dysbiosis in IBD.

\section{Abbreviations}

CD: Crohn's disease; DAl: Disease activity index; DSS: Dextran sulfate sodium; IBD: Inflammatory bowel disease; OTUs: Operational taxonomic units; PCoA: Principal coordinate analysis; SQE: Sasa quelpaertensis leaf extract; SSZ: Sulfasalazine; UC: Ulcerative colitis

\section{Acknowledgements}

We would like to express our thanks to Kyung-Mi Kim for helping with the animal experiment and Hee-Chul Ko for providing Sasa quelpaertensis Nakai leaves extract.

\section{Funding}

This work was supported by the "Cooperative Research Program for Agriculture Science \& Technology Development (Project No, PJ009777)" Rural Development Administration, Republic of Korea; and the Brain Korea 21 Plus (Project No. 22A20130012143).

\section{Availability of data and materials}

The data and materials of this article are included within the article.

\section{Authors' contributions}

YY performed experiments, data analyses, and prepared the first draft of the manuscript. BSK and $\mathrm{K}$ have assisted in the conception of the study and analysis of the data. YK designed the experiments, provided the reagents/ analysis and prepared final manuscript. All authors read and approved the final manuscript.

\section{Competing interests}

The authors declare that they have no competing interests.

\section{Consent for publication}

Not applicable.

\section{Ethics approval and consent to participate}

The study protocol involving the use of animals in the present study was approved by the Animal Care and Use Committee of Ewha Womans University (IACUC approval no: IACUC 14-070).

\section{Author details}

'Department of Nutritional Science and Food Management, Ewha Womans University, Seoul 03760, Republic of Korea. ${ }^{2}$ Department of Life Science, Hallym University, Chuncheon, Gangwon-do 24252, Republic of Korea. ${ }^{3}$ Department of Biology, Jeju National University, Jejusi, Jeju 63243, Republic of Korea.

Received: 28 June 2016 Accepted: 1 November 2016

Published online: 25 November 2016

\section{References}

1. Keyashian K, Annunziata ML, Sakuraba A, Hanauer S. Management of inflammatory bowel disease: past, present and future. Expert Rev Clin Immunol. 2012;8:303-5.

2. Selinger $\mathrm{CP}$, Leong RW. Mortality from inflammatory bowel diseases. Inflamm Bowel Dis. 2012;18:1566-72.

3. Sartor RB, Mazmanian SK. Intestinal Microbes in Inflammatory Bowel Diseases. Am J Gastroenterol Suppl. 2012;1:15-21.
4. Savage DC. Microbial ecology of the gastrointestinal tract. Annu Rev Microbiol. 1977;31:107-33.

5. Penders J, Thijs C, Vink C, Stelma FF, Snijders B, Kummeling I, et al. Factors influencing the composition of the intestinal microbiota in early infancy. Pediatrics. 2006;118:511-21.

6. Turnbaugh PJ, Ley RE, Hamady M, Fraser-Liggett CM, Knight R, Gordon Jl. The human microbiome project. Nature. 2007;449:804-10.

7. Louis P, Hold GL, Flint HJ. The gut microbiota, bacterial metabolites and colorectal cancer. Nat Rev Microbiol. 2014;12:661-72.

8. Ussar S, Griffin NW, Bezy O, Fujisaka S, Vienberg S, Softic S, et al. Interactions between Gut Microbiota, Host Genetics and Diet Modulate the Predisposition to Obesity and Metabolic Syndrome. Cell Metab. 2015;22:516-30

9. Tremaroli $V$, Backhed F. Functional interactions between the gut microbiota and host metabolism. Nature. 2012:489:242-9.

10. Hur KY, Lee MS. Gut Microbiota and Metabolic Disorders. Diabetes Metab J. 2015;39:198-203.

11. Karlsson F, Tremaroli V, Nielsen J, Backhed F. Assessing the human gut microbiota in metabolic diseases. Diabetes. 2013:62:3341-9.

12. Galland L. The gut microbiome and the brain. J Med Food. 2014;17:1261-72.

13. Trompette A, Gollwitzer ES, Yadava K, Sichelstiel AK, Sprenger N, Ngom-Bru $C$, et al. Gut microbiota metabolism of dietary fiber influences allergic airway disease and hematopoiesis. Nat Med. 2014;20:159-66.

14. Duenas M, Munoz-Gonzalez I, Cueva C, Jimenez-Giron A, Sanchez-Patan F, Santos-Buelga C, et al. A survey of modulation of gut microbiota by dietary polyphenols. Biomed Res Int. 2015;2015:850902.

15. Boeing $H$, Bechthold A, Bub A, Ellinger S, Haller D, Kroke A, et al. Critical review: vegetables and fruit in the prevention of chronic diseases. Eur J Nutr. 2012;51:637-63.

16. Kang SW, Kang SI, Shin HS, Yoon SA, Kim JH, Ko HC, et al. Sasa quelpaertensis Nakai extract and its constituent p-coumaric acid inhibit adipogenesis in 3 T3-L1 cells through activation of the AMPK pathway. Food Chem Toxicol. 2013;59:380-5.

17. Kim KM, Kim YS, Lim JY, Min SJ, Shin JH, Ko HC, et al. Sasa quelpaertensis leaf extract suppresses dextran sulfate sodium-induced colitis in mice by inhibiting the proinflammatory mediators and mitogen-activated protein kinase phosphorylation. Nutr Res. 2014;34:894-905.

18. Min SJ, Lim JY, Kim HR, Kim SJ, Kim Y. Sasa quelpaertensis Leaf Extract Inhibits Colon Cancer by Regulating Cancer Cell Stemness in Vitro and in Vivo. Int J Mol Sci. 2015;16:9976-97.

19. Kim KM, Kim YS, Lim JY, Min SJ, Ko HC, Kim SJ, et al. Intestinal antiinflammatory activity of Sasa quelpaertensis leaf extract by suppressing lipopolysaccharide-stimulated inflammatory mediators in intestinal epithelial Caco-2 cells co-cultured with RAW 264.7 macrophage cells. Nutr Res Pract. 2015;9:3-10.

20. Kim JH, Kang SI, Shin HS, Yoon SA, Kang SW, Ko HC, et al. Sasa quelpaertensis and p-coumaric acid attenuate oleic acid-induced lipid accumulation in HepG2 cells. Biosci Biotechnol Biochem. 2013;77:1595-8.

21. Jeon YS, Chun J, Kim BS. Identification of household bacterial community and analysis of species shared with human microbiome. Curr Microbiol. 2013;67:557-63.

22. Kim OS, Cho YJ, Lee K, Yoon SH, Kim M, Na H, et al. Introducing EzTaxon-e: a prokaryotic $16 \mathrm{~S}$ rRNA gene sequence database with phylotypes that represent uncultured species. Int I Syst Evol Microbiol. 2012;62:716-21.

23. Edgar RC, Haas BJ, Clemente JC, Quince C, Knight R. UCHIME improves sensitivity and speed of chimera detection. Bioinformatics. 2011;27: 2194-200.

24. Schloss PD, Westcott SL, Ryabin T, Hall JR, Hartmann M, Hollister EB, et al. Introducing mothur: open-source, platform-independent, communitysupported software for describing and comparing microbial communities. Appl Environ Microbiol. 2009;75:7537-41.

25. Hamady M, Lozupone C, Knight R. Fast UniFrac: facilitating high-throughput phylogenetic analyses of microbial communities including analysis of pyrosequencing and PhyloChip data. ISME J. 2010;4:17-27.

26. Sanchez-Fidalgo S, Cardeno A, Sanchez-Hidalgo M, Aparicio-Soto M, de la Lastra CA. Dietary extra virgin olive oil polyphenols supplementation modulates DSS-induced chronic colitis in mice. J Nutr Biochem. 2013; 24:1401-13.

27. Walker AW, Sanderson JD, Churcher C, Parkes GC, Hudspith BN, Rayment N, et al. High-throughput clone library analysis of the mucosa-associated microbiota reveals dysbiosis and differences between inflamed and non- 
inflamed regions of the intestine in inflammatory bowel disease. BMC Microbiol. 2011;11:7.

28. Morgan XC, Tickle TL, Sokol H, Gevers D, Devaney KL, Ward DV, et al. Dysfunction of the intestinal microbiome in inflammatory bowel disease and treatment. Genome Biol. 2012;13:R79.

29. Sartor RB. Microbial influences in inflammatory bowel diseases. Gastroenterology. 2008;134:577-94.

30. Casellas F, Borruel N, Papo M, Guarner F, Antolin M, Videla S, et al. Antiinflammatory effects of enterically coated amoxicillin-clavulanic acid in active ulcerative colitis. Inflamm Bowel Dis. 1998:4:1-5.

31. Gkouskou KK, Deligianni C, Tsatsanis C, Eliopoulos AG. The gut microbiota in mouse models of inflammatory bowel disease. Front Cell Infect Microbiol. 2014;4:28.

32. Berry D, Schwab C, Milinovich G, Reichert J, Ben Mahfoudh K, Decker T, et al. Phylotype-level 16S rRNA analysis reveals new bacterial indicators of health state in acute murine colitis. ISME J. 2012;6:2091-106.

33. Wohlgemuth S, Haller D, Blaut M, Loh G. Reduced microbial diversity and high numbers of one single Escherichia coli strain in the intestine of colitic mice. Environ Microbiol. 2009;11:1562-71.

34. Yeom Y, Kim Y. The Sasa quelpaertensis Leaf Extract Inhibits the Dextran Sulfate Sodium-induced Mouse Colitis Through Modulation of Antioxidant Enzyme Expression. J Cancer Prev. 2015;20:136-46.

35. Osada T, Ohkusa T, Okayasu I, Yoshida T, Hirai S, Beppu K, et al. Correlations among total colonoscopic findings, clinical symptoms, and laboratory markers in ulcerative colitis. J Gastroenterol Hepatol. 2008;23:S262-7.

36. Arab HH, Al-Shorbagy MY, Abdallah DM, Nassar NN. Telmisartan attenuates colon inflammation, oxidative perturbations and apoptosis in a rat model of experimental inflammatory bowel disease. PLoS One. 2014;9, e97193.

37. Winter SE, Lopez CA, Baumler AJ. The dynamics of gut-associated microbial communities during inflammation. EMBO Rep. 2013;14:319-27.

38. Frank DN, St Amand AL, Feldman RA, Boedeker EC, Harpaz N, Pace NR. Molecular-phylogenetic characterization of microbial community imbalances in human inflammatory bowel diseases. Proc Natl Acad Sci U S A. 2007;104:13780-5.

39. Ott SJ, Plamondon S, Hart A, Begun A, Rehman A, Kamm MA, et al. Dynamics of the mucosa-associated flora in ulcerative colitis patients during remission and clinical relapse. J Clin Microbiol. 2008:46:3510-3.

40. Martinez C, Antolin M, Santos J, Torrejon A, Casellas F, Borruel N, et al. Unstable composition of the fecal microbiota in ulcerative colitis during clinical remission. Am J Gastroenterol. 2008;103:643-8.

41. Hooper LV, Littman DR, Macpherson AJ. Interactions between the microbiota and the immune system. Science. 2012;336:1268-73.

42. Clemente JC, Ursell LK, Parfrey LW, Knight R. The impact of the gut microbiota on human health: an integrative view. Cell. 2012;148:1258-70.

43. Nguyen TLA, Vieira-Silva S, Liston A, Raes J. How informative is the mouse for human gut microbiota research? Dis Model Mech. 2015;8:1-16.

44. Nell S, Suerbaum S, Josenhans $C$. The impact of the microbiota on the pathogenesis of IBD: lessons from mouse infection models. Nat Rev Microbiol. 2010;8:564-77.

45. Samuel BS, Shaito A, Motoike T, Rey FE, Backhed F, Manchester JK, et al. Effects of the gut microbiota on host adiposity are modulated by the shortchain fatty-acid binding G protein-coupled receptor, Gpr41. Proc Natl Acad Sci U S A. 2008;105:16767-72.

46. Ebinuma H, Nanto K, Kasahara S, Komamine A. Marker-free gene targeting by recombinase-mediated cassette exchange. Methods Mol Biol. 2012;847: 379-90.

47. Peterson DA, Frank DN, Pace NR, Gordon Jl. Metagenomic approaches for defining the pathogenesis of inflammatory bowel diseases. Cell Host Microbe. 2008;3:417-27.

48. Reichardt N, Duncan SH, Young P, Belenguer A, McWilliam Leitch C, Scott $\mathrm{KP}$, et al. Phylogenetic distribution of three pathways for propionate production within the human gut microbiota. ISME J. 2014;8:1323-35.

49. Grider JR, Piland BE. The peristaltic reflex induced by short-chain fatty acids is mediated by sequential release of 5-HT and neuronal CGRP but not BDNF. Am J Physiol Gastointest Liver Physiol. 2007;292:G429-37.

50. Lupp C, Robertson ML, Wickham ME, Sekirov I, Champion OL, Gaynor EC, et al. Host-mediated inflammation disrupts the intestinal microbiota and promotes the overgrowth of Enterobacteriaceae. Cell Host Microbe. 2007;2:119-29.

51. Atarashi K, Tanoue T, Oshima K, Suda W, Nagano Y, Nishikawa H, et al. Treg induction by a rationally selected mixture of Clostridia strains from the human microbiota. Nature. 2013;500:232-6.
52. Ridlon JM, Kang DJ, Hylemon PB. Bile salt biotransformations by human intestinal bacteria. J Lipid Res. 2006;47:241-59.

53. Malar DS, Devi KP. Dietary polyphenols for treatment of Alzheimer's diseasefuture research and development. Curr Pharm Biotechnol. 2014;15:330-42.

54. Williamson G, Manach C. Bioavailability and bioefficacy of polyphenols in humans. II. Review of 93 intervention studies. Am J Clin Nutr. 2005;81:243s-55s.

55. Selma MV, Espin JC, Tomas-Barberan FA. Interaction between phenolics and gut microbiota: role in human health. J Agric Food Chem. 2009;57:6485-501.

56. Rastmanesh R. High polyphenol, low probiotic diet for weight loss because of intestinal microbiota interaction. Chem Biol Interact. 2011;189:1-8.

57. Cueva C, Sanchez-Patan F, Monagas M, Walton GE, Gibson GR, MartinAlvarez PJ, et al. In vitro fermentation of grape seed flavan-3-ol fractions by human faecal microbiota: changes in microbial groups and phenolic metabolites. FEMS Microbiol Ecol. 2013;83:792-805.

58. Rosillo MA, Sanchez-Hidalgo M, Cardeno A, de la Lastra CA. Protective effect of ellagic acid, a natural polyphenolic compound, in a murine model of Crohn's disease. Biochem Pharmacol. 2011;82:737-45.

59. Sartor RB. Therapeutic manipulation of the enteric microflora in inflammatory bowel diseases: antibiotics, probiotics, and prebiotics. Gastroenterology. 2004;126:1620-33.

\section{Submit your next manuscript to BioMed Central and we will help you at every step:}

- We accept pre-submission inquiries

- Our selector tool helps you to find the most relevant journal

- We provide round the clock customer support

- Convenient online submission

- Thorough peer review

- Inclusion in PubMed and all major indexing services

- Maximum visibility for your research

Submit your manuscript at www.biomedcentral.com/submit
Biomed Central 